\title{
Matrix-Assisted Pulsed Laser Thin Film Deposition by Using Nd:YAG Laser
}

\author{
Francesco Bloisi, Mario Barra, Antonio Cassinese, and Luciano Rosario Maria Vicari
}

CNR-SPIN and Department of Physics Science, University of Naples, Piazzale Tecchio 80, 80125 Naples, Italy

Correspondence should be addressed to Antonio Cassinese, cassinese@na.infn.it

Received 25 January 2012; Accepted 29 March 2012

Academic Editor: Sevan P. Davtyan

Copyright () 2012 Francesco Bloisi et al. This is an open access article distributed under the Creative Commons Attribution License, which permits unrestricted use, distribution, and reproduction in any medium, provided the original work is properly cited.

\begin{abstract}
Matrix-Assisted Pulsed Laser Evaporation (MAPLE) is a deposition technique, developed from Pulsed Laser Deposition (PLD) especially well suited for producing organic/polymeric thin films, which can take advantage from using Nd:YAG laser. Depending on the relative values of light absorption coefficients of the solvent and of the molecules to be deposited, laser energy is directly absorbed by the solvent or is transferred to it, providing a softer desorption mechanism with respect to PLD. In PLD ultraviolet laser radiation is commonly used, but in MAPLE, since easily damaged molecules are usually involved, the use of Nd:YAG laser offers the advantage to allow selecting laser wavelength from ultraviolet $(266 \mathrm{~nm}$ or $355 \mathrm{~nm}$, corresponding to $4.66 \mathrm{eV}$ or $3.49 \mathrm{eV}$ photon energies, resp.) to visible $(532 \mathrm{~nm}, 2.33 \mathrm{eV})$ to infrared $(1064 \mathrm{~nm}, 1.17 \mathrm{eV})$. In this paper, the MAPLE technique is described in details, together with a survey of current and possible future applications for both organic and biomaterial deposition taking into account the advantages of using an Nd:YAG laser. Beside other results, we have experimental confirmation that MAPLE applications are not limited to transparent molecules highly soluble in light absorbing solvent, thus allowing deposition of poorly soluble light absorbing molecules suspended in a light transparent liquid.
\end{abstract}

\section{Introduction}

Matrix-Assisted Pulsed Laser Evaporation (MAPLE) [1] is a thin film deposition technique derived from Pulsed Laser Deposition (PLD) with the aim to make possible the deposition of soft (e.g., polymeric, organic, bio) materials. Indeed, despite PLD is nowadays successfully used to produce highquality thin films [2], it generally fails when soft materials [3] are considered since the laser beam energy used for target ablation damages such molecules. Conversely, in the MAPLE approach, the target is a frozen solution or suspension of the guest material in a matrix. The term laser evaporation contained in the acronym suggests a guest extraction technique more gentle than laser ablation. Experimental works [4-7] and simulation studies $[8,9]$, however, have shown that the initially proposed evaporation model $[1,3,10]$ where laser energy is mainly absorbed by the matrix which evaporates and transfers thermal energy to the guest molecules, being, on their turn, gently desorbed, is not adequate in describing the complex physical process involved in the ablation of such a composite target.

As a consequence, the two conditions originally assumed to be crucial in order to obtain a MAPLE deposition (i.e. the guest material must be highly soluble in the matrix and the laser radiation must be mainly absorbed by the matrix and negligibly absorbed by the guest material) can be relaxed. Several authors have, indeed, experimentally shown that both such conditions are not strictly necessary in order to successfully deposit a thin film of the guest material: MAPLE technique has been used to deposit matrix suspended nanoparticles $[12,13]$ and light absorbing molecules in a transparent solvent $[11,14]$. Given these results, MAPLE deposition should be assumed being characterized only by (a) the presence of a matrix in the target and (b) the absence (or at least a significant reduction with respect to classical PLD technique) of laser-induced damage to guest molecules.

The good results reported, in recent years, by many authors in depositing polymers, biomaterials, and organic 
molecules for an increasing number of applications $[6,15-$ 18] justify the large interest in both better understanding the guest extraction mechanism [19] and determining the effect of several deposition parameters [20-23] (guest/matrix volume fraction, boiling temperature and vapour pressure of matrix, guest damage temperature, laser pulse energy and repetition rate, laser radiation penetration depth, substrate temperature during deposition, etc.) on the MAPLEdeposited film characteristics.

Another important parameter concerning the MAPLE deposition is the wavelength (or photon energy) of the laser light used for target ablation/evaporation. It must be chosen in order to avoid damages to guest molecules (IR radiation corresponds to low-energy photons) but must be absorbed by matrix (most common solvents are almost transparent to IR radiation while they absorb UV radiation). In most MAPLE deposition experiments $\mathrm{KrF}$ (emission at $248 \mathrm{~nm}$, UV, $5.00 \mathrm{eV}$ ) or ArF (emission at $193 \mathrm{~nm}, \mathrm{UV}, 6.42 \mathrm{eV}$ ) lasers or an Nd:YAG laser equipped with Fourth Harmonic Generation (FHG) device (final emission at $266 \mathrm{~nm}$, UV, $4.66 \mathrm{eV}$ ) is used as energy source for target ablation. However the use of an Nd:YAG laser has the additional advantage to allow selection of longer wavelengths (VIS or IR) and therefore lower photon energies. The use of lower-energy VIS or IR photons can be advantageous since it reduces damaging actions on soft molecules but has the inconvenience that most solvents are almost transparent to VIS-IR radiation.

Here we summarize the results obtained with a MAPLE deposition system which uses an Nd:YAG laser equipped with Second Harmonic Generation (SHG) and Third Harmonic Generation (THG), in growing thin films for organic electronics (polythiophene, eumelanin) and for biomedical applications (PEG, DOPA, PDLLA). It must be remarked that our experiments confirm that MAPLE applications must not be limited to light transparent molecules highly soluble in light absorbing solvent but can be successfully performed for suspensions of light absorbing molecules (or even molecular aggregates or nanoparticles) in a light transparent liquid.

\section{The MAPLE Deposition Technique}

A typical MAPLE deposition system [24] is sketched in Figure 1 and shown in Figure 2. The target is a frozen (usually at liquid nitrogen temperature) dilute (usually less than few $\mathrm{wt} \%$ ) solution of the delicate material in a volatile solvent. The pulsed laser beam impinging on the target removes from its surface both the light and small solvent molecules and the heavy and large solute ones, but only the latter deposit onto the substrate, placed in proximity of the target, at a distance of few millimeters. On the contrary, solvent molecules are pumped away by the vacuum system. The presence of the matrix (the solvent) eliminates or at least minimizes the photochemical damage of the delicate molecules.

In a first description [3] (assuming that laser radiation is mostly absorbed by the solvent) it was supposed that the solute molecules gain enough kinetic energy, through collective collisions with the evaporating solvent molecules, to be transferred into the gas phase with a soft desorption mechanism.

In some cases [1] thin films deposited with MAPLE technique have shown higher surface uniformity than films deposited with other techniques, but general experimental evidence [5-7, 25] shows significant surface roughness [4] or curious "deflated balloon" features [26].

(Molecular Dynamics) MD simulations [8,9] carried out in order to explain the observed film surface characteristics have shown that the "evaporation" mechanism is rather complex.

The laser beam overheats, up to the limit of its thermodynamic stability, a small surface region of the target. The decomposition of the target surface generates a structure of interconnected liquid regions.

Subsequently such a foamy structure aggregates into a mixture of liquid droplets and gas-phase matrix molecules. Both small (and fast) and large (and slow) clusters generated during the ablation of the MAPLE target are therefore present in the material ejected from the target.

This "matrix/polymer clusters" [8] ejection mechanism provides a valid explanation of the "deflated balloon" surface features and of the surface roughness experimentally observed.

Simulations have been carried out for nonabsorbing solute molecules in an absorbing matrix, but a similar mechanism may take place for absorbing molecules dispersed in a transparent solvent.

In MAPLE depositions we must distinguish between two different situations depending on the relative values of the absorption coefficients of the solute and of the solvent for the selected laser radiation. If the laser energy is mostly absorbed by the frozen solvent, its local abrupt evaporation causes delicate solute molecules to be ejected from target. If, on the contrary, the solvent is almost transparent to laser radiation, the laser energy is mainly absorbed by the solute molecules, but, due to their low concentration, we have a highly nonhomogeneous light absorption. Laser evaporation of a frozen dilute solution of an absorbing solute in a transparent solvent can be assumed similar to laser ablation of a solution with spatially heterogeneous absorption [27]: the ablation efficiency of the heterogeneous solution is higher by more than one order of magnitude with respect to homogeneous absorption (as in PLD). This implies that MAPLE, with respect to $\mathrm{PLD}$, allows reducing laser pulse energy by one order of magnitude, thus greatly reducing the negative effects of laser irradiation.

In any case, compared to PLD, the MAPLE technique results in a more soft mechanism of delicate material (complex polymeric/organic molecules or biomaterials or nanostructures) transfer from target to substrate.

This means that MAPLE deposition technique is suitable for several situations in which other techniques (spin coating, drop casting, etc.) and especially PLD deposition cannot be used, but, on the other side, it requires a fine adjustment of the deposition layout (solvent choice, solute concentration, substrate position, and orientation) and parameters (laser radiation wavelength, beam fluence, pulse duration, pulse repetition rate, substrate temperature). 


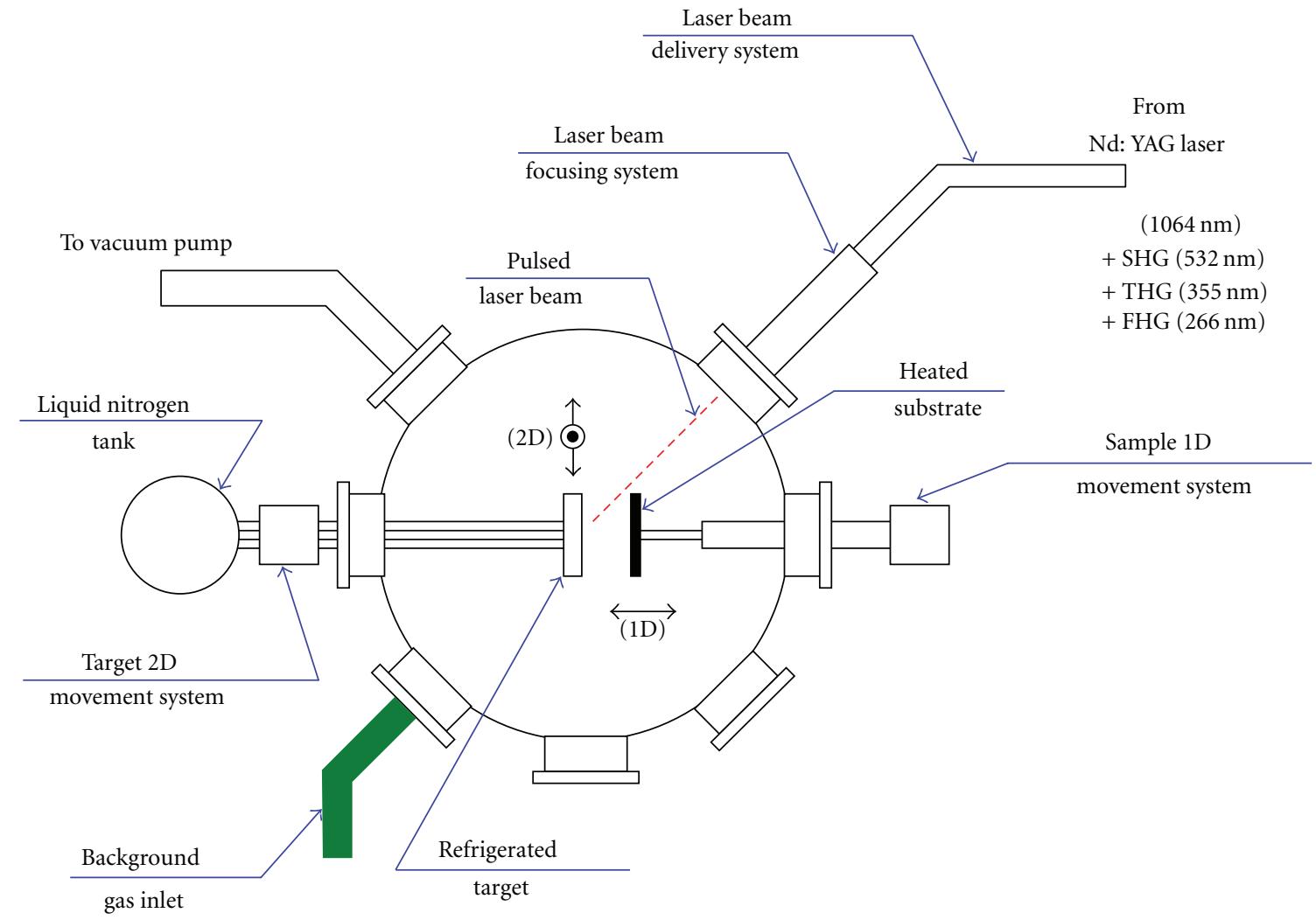

Figure 1: Sketch of the MAPLE deposition system shown in Figure 2.

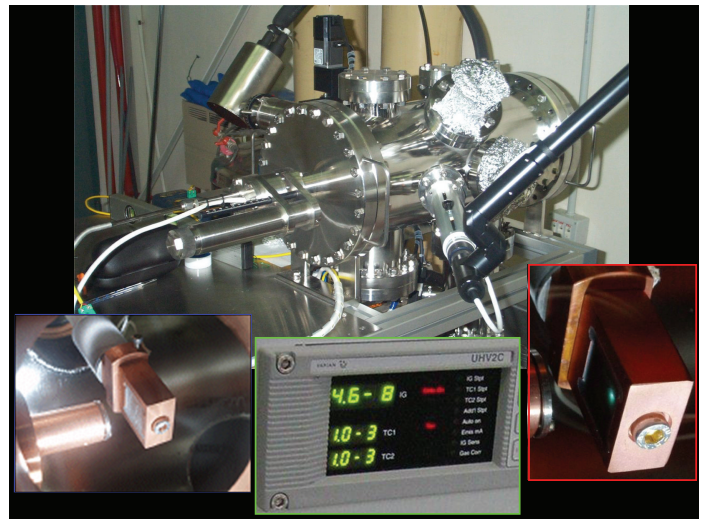

Figure 2: A MAPLE deposition system. First inset shows the substrate holder (on the left) and the target holder (on the right) inside vacuum chamber. Second inset shows a detail of the control panel. Third inset shows the laser irradiation of the target during a MAPLE deposition.

In order to get higher flexibility in the laser wavelength choice, the Nd:YAG laser offers the possibility to use longer wavelengths $(266,355,532,1064 \mathrm{~nm})$ and therefore lower energies $(4.66,3.49,2.33,1.17 \mathrm{eV})$ with respect to $\mathrm{KrF}$ $(248 \mathrm{~nm}, 5.00 \mathrm{eV})$ or $\operatorname{ArF}(193 \mathrm{~nm}, 6.41 \mathrm{eV})$ lasers.

\section{MAPLE Applications}

MAPLE technique founds a wide range of applications including deposition of polymeric thin films for optoelectronics and for chemical sensors and deposition.

A broad range of MAPLE applications include the deposition of polymeric thin films for optoelectronics and chemical sensors $[6,10,15,28,29]$, growth of active protein thin films [5, 30-33], polymer-carbon nanotube composites $[25,34]$, and films containing nanoparticles [35].

We have obtained interesting results using Nd:YAG/ MAPLE system for deposition of materials of interest in biomedical (PEG, DOPA, PDLLA) and organic-electronics (polythiophene, eumelanin) applications.

\subsection{Biomaterials}

3.1.1. PEG. Polyethylene glycol (PEG) is a polymer with technologically important applications as a biomaterial (material interfacing with living tissues or biological fluids): tissue engineering, spatial patterning of cells, antibiofouling (biofouling is the cellular and proteinaceous adhesion on biomaterial surface and is commonly avoided through the immobilization of antifouling polymers on the surface to protect), and biocompatible coatings (in drug delivery coatings or orthopaedic implants). Thin films of high quality 


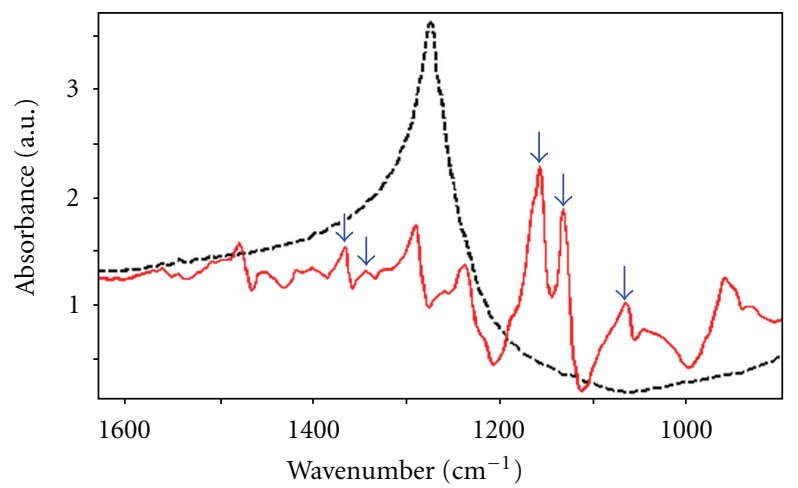

(a)

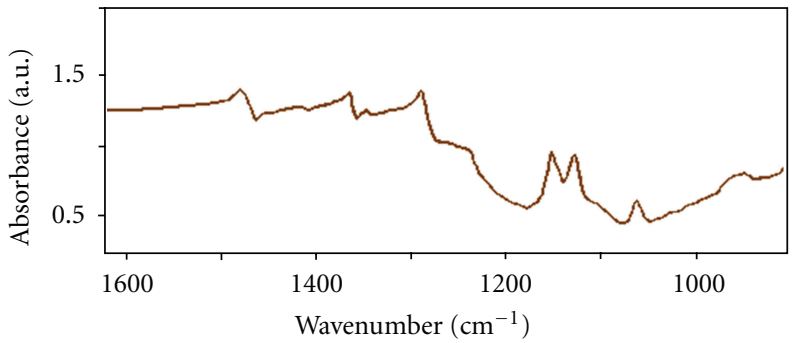

(b)

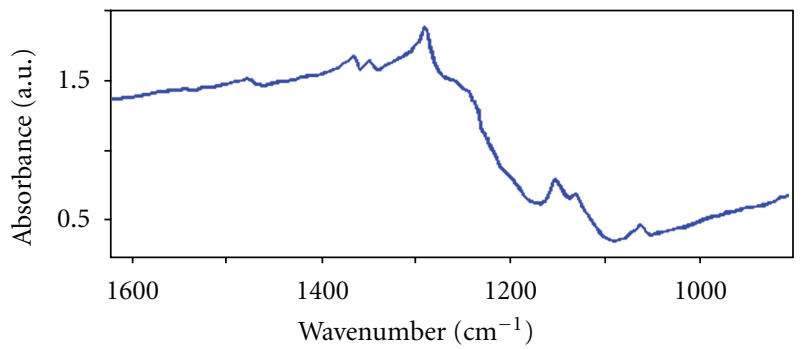

(c)

FIGURE 3: (a) Reference IR spectra, performed in reflectance mode, in the range $900-1600 \mathrm{~cm}^{-1}$ of the substrate (a glass slide) without (black dashed line) and with a dried drop of PEG (red full line). The arrows show the $\mathrm{C}-\mathrm{O}-\mathrm{C}$ aliphatic ether antisymmetric stretching vibration bands in the range $1150-1060 \mathrm{~cm}^{-1}$ and the $\mathrm{C}-\mathrm{OH}$ deformation vibration absorption in the range $1400-1300 \mathrm{~cm}^{-1}$. (b) and (c) show typical spectra carried out on PEG films deposited on glass slides with MAPLE technique using laser radiation at $532 \mathrm{~nm}$ and $355 \mathrm{~nm}$, respectively.

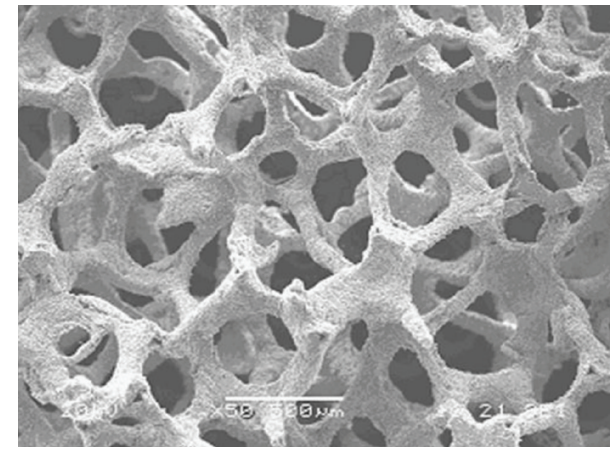

FIGURE 4: SEM image showing the typical porous structure of a bioglass-based glass-ceramic scaffolds (reproduced with permission from $[11])$.

are required and the deposition of a film with the same chemical and structural properties of bulk PEG is essential.

Due to its high solubility in water (but also in other common solvents) and its availability in a wide range of molecular weights (from less than $10^{3} \mathrm{~g} / \mathrm{mol}$ to about $\left.10^{7} \mathrm{~g} / \mathrm{mol}\right)$, PEG is often used as a test molecule for organic/ polymeric thin film depositions.

The KrF laser (UV radiation at $248 \mathrm{~nm}$ ) is widely used for PLD, however its radiation potentially damages most organic compounds and polymers (e.g., phenyl rings, pervasive in organic chemistry, absorb radiation at $250 \mathrm{~nm}$ ). MAPLE technique has already been carried out for PEG thin films deposition using ArF laser (UV radiation at $193 \mathrm{~nm}$ ) [36], but other researches have tried to use lasers involving longer wavelengths, such as Er:YAG (IR radiation at $2937 \mathrm{~nm}$ ) [20] or Nd:YAG (third harmonic UV radiation at $355 \mathrm{~nm}$ ) [7].

We have recognized, by comparison of the reflectance mode IR spectra of deposited films (Figure 3), that similar results can be also obtained with visible (Nd:YAG second harmonic at $532 \mathrm{~nm}$ ) [16] laser radiation.

In most biomedical applications an important issue is the requirement for a mechanism to anchor PEG on the substrate. This is sometimes achieved with polymer functionalization, but at the cost of changing molecular structure. MAPLE is essentially a solvent-free deposition technique (the solvent used as matrix in target does not reaches the substrate) and this easily allows the interposition of an adhesion layer, for example, 3-(3,4-dihydroxyphenyl)-2-methylL-alanine (m-DOPA) [37].

3.1.2. PDLLA. Tissue engineering and regenerative medicine are important area of research involving a multitude of disciplines, including cell biology, biomaterials science, and characterization of surfaces and cell material interactions. A common tissue engineering strategy uses bioresordable scaffolds made of engineered biomaterials in order to give 


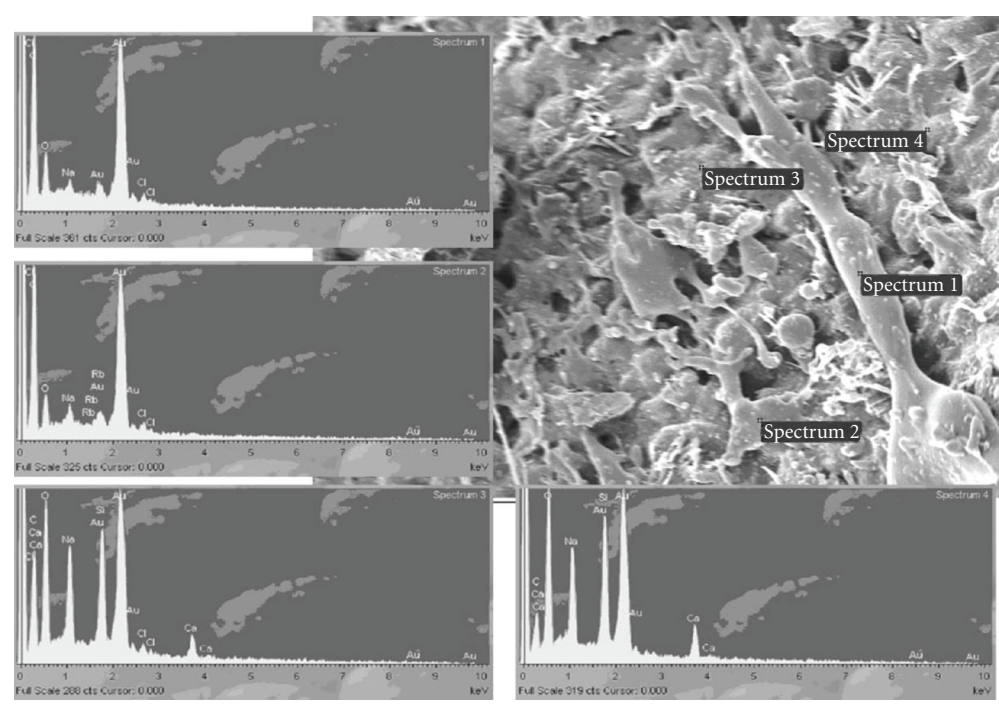

FIGURE 5: SEM image of the bioglass scaffold after MAPLE deposition of a PDLLA coating and EDX analyses carried out in different places show that porous structure is partially preserved (reproduced with permission from [11]).

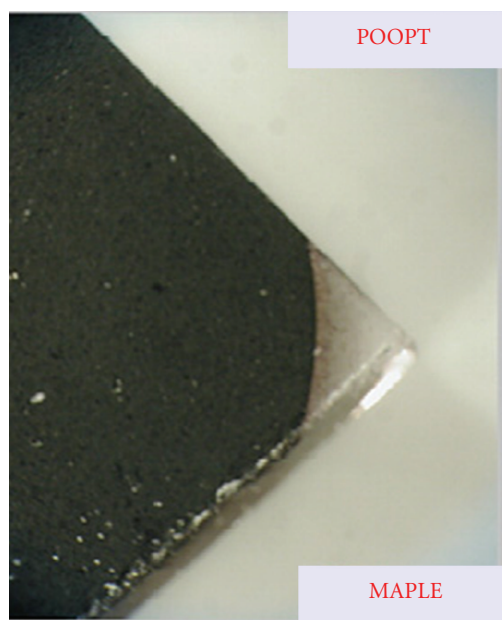

FIgURE 6: POOPT thin film deposited on a glass slide with MAPLE technique with the following layout/deposition parameters: target: POOPT $0.56 \mathrm{wt} . \%$ in chloroform; target temperature: $-187^{\circ} \mathrm{C}$; pressure: $2.310^{-7}$ Torr; laser wavelength: $532 \mathrm{~nm}$; pulse repetition rate: 10 pulse/s; pulse duration: $6 \mathrm{~ns}$; pulse energy: $68 \mathrm{~mJ}$; beam spot size: $0.5 \mathrm{~cm}^{2}$; total pulses: 20000; scanned target area: $2 \mathrm{~cm}^{2}$.

a temporary mechanical support for natural tissue regeneration.

Bone regeneration requires scaffolds with adequate properties from both the biological and mechanical points of view: high bioactivity (the ability to react with physiological fluids and form tenacious bonds to tissues through cellular activity) and bioresorbability (the ability to biodegrade), interconnected porosity to allow cell growth, and sufficient hardness to avoid collapsing during handling and after implantation.

The 4555 Bioglass is a commercially available material [38] whose main advantage is its high bioactivity. Bioglass scaffolds produced using the foam replica technique [39] are good candidates for bone regeneration. However, the resulting bioglass-based glass-ceramic scaffolds (Figure 4) are very brittle. Several approaches, mainly combining it with synthetic polyesters, such as Poly(D,L-lactide) (PDLLA), have been proposed in order to improve the mechanical properties of brittle bioactive glass scaffolds for bone tissue engineering. However the polymeric coating cannot be obtained by immersion since it must maintain the porosity of the structure. This requirement has induced us to use the MAPLE deposition technique in order to obtain a graded structure with a core made fully of bioglass and an outer layer made of the polymer-coated bioglass $[11,18]$. The SEM image of the surface of a PDLLA-coated bioglass scaffold (Figure 5) and the EDX analysis carried out in several spots (insets in the same figure) shows that deposition is discontinuous forming discrete fibrils covering partially the surface of the pores. Since fibrils penetrate the pores, they are expected to provide a bridging element to impede the propagation of cracks, thus improving mechanical properties of scaffold structure.

3.2. Polythiophene. Semiconducting properties of conjugated polymers are due to the high extent of $\pi$ orbitals delocalisation, thus forming a valence band of overlapping $\pi$ bonds and a conduction band of overlapping $\pi^{*}$ antibonding orbitals. Within polyconjugated systems, substituted polythiophenes spin-coated films have given interesting results for its conductive properties $[40,41]$. Charge mobility is affected by both polymer conjugation length and chain packing, so that highly regioregular polymers are required. We have successfully used MAPLE technique in order to deposit regio-regular $\mathrm{H}-\mathrm{T}$ poly [3-(4-octyloxyphenyl)thiophene] (POOPT) thin films on glass (Figure 6) and $\mathrm{KBr}$ substrates $[24,42]$ and examined the effect of substrate temperature on the conformation and structure of the films [21]. FTIR 


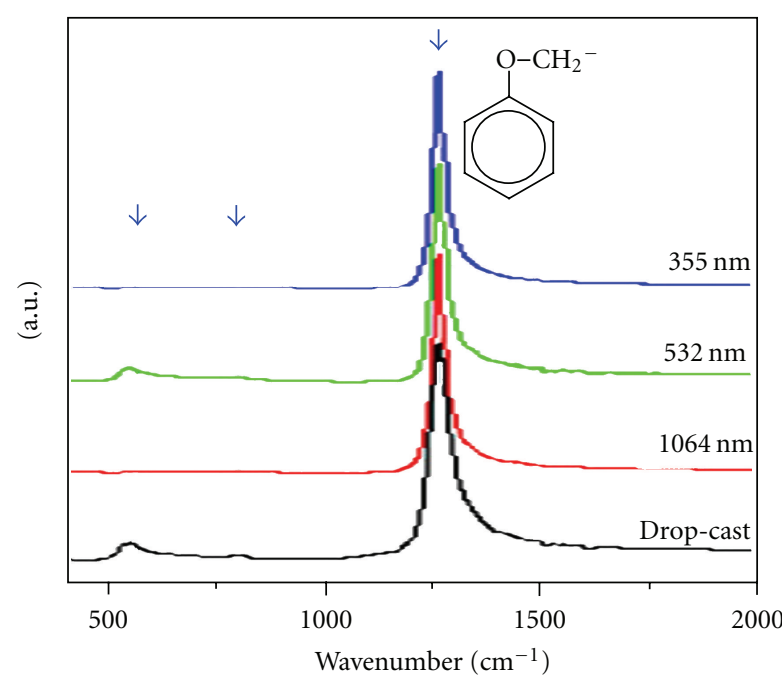

(a)

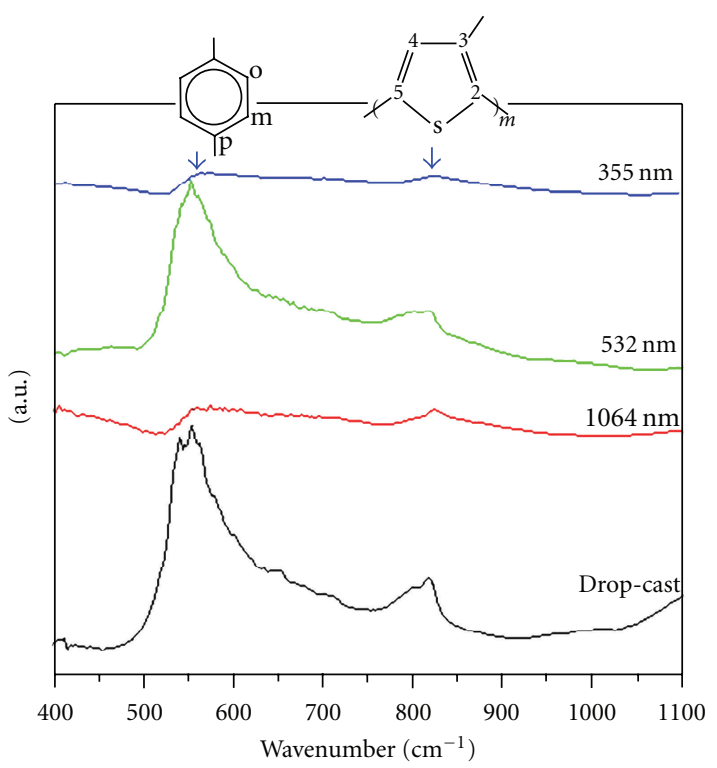

(b)

FIGURE 7: DRIFT-IR spectra of POOPT drop cast and MAPLE deposited using different laser radiation wavelength (IR: $1064 \mathrm{~nm}$, VIS: $532 \mathrm{~nm}$, and UV: $355 \mathrm{~nm}$ ). The shape and intensity of the $1270 \mathrm{~cm}^{-1}$ band suggest a highly regular disposition of the polymer side chains and, consequently, of the polymer backbone. The lowering of the bands at $560 \mathrm{~cm}^{-1}$ and at $806 \mathrm{~cm}^{-1}$ in films deposited using IR or UV laser radiation suggests that in these films some decomposition phenomena occurred, likely at the polymer backbone-side chain level.

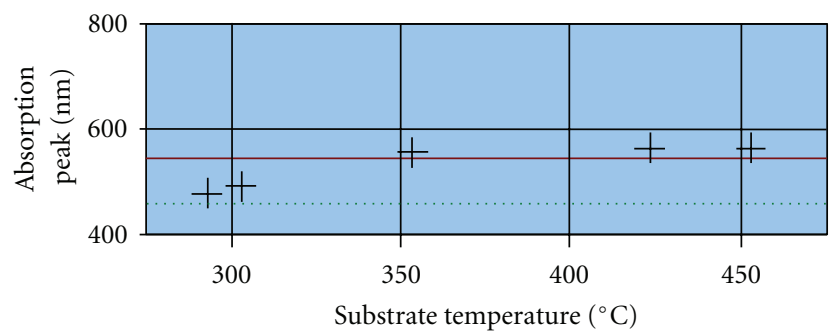

FIGURE 8: The position of the absorption peak in the visible region of the spectrum is plotted versus substrate temperature during MAPLE deposition. Full line represents peak value for spin-coated films, while dashed line represents peak value for a POOPT solution in chloroform.

analysis (Figure 7) suggests that laser radiation at $532 \mathrm{~nm}$ is the better choice for MAPLE deposition of POOPT films.

As observed before, MAPLE deposition technique often leads to a cluster deposition, but it is also well known that the temperature of the substrate during film deposition is able to change the morphology and other film characteristics.

UV-Vis spectroscopy gives information about films conformation and packing since the position of the absorption peak in the visible region is affected by the polymer steric conformation and suggests an increase of its planarization due to simultaneous decrease of the random coil conformation and increase of untwisted configuration.

Our main result (Figure 8) is the observation that the polymer conjugation length increases with increasing substrate temperature during deposition, and at about 150$180^{\circ} \mathrm{C}$, it is slightly higher than the one obtained for spin coated films; substrate temperature increase also promotes side-chain commutation to different steric configurations.

3.3. Eumelanin. Eumelanin is an important pigment almost ubiquitous in animals and plants exhibiting interesting charge transport capabilities, but its poor solubility in common solvents represents a severe limitation for the realization of thin films.

As observed previously, MAPLE deposition technique can be successfully performed starting from a frozen suspension. Since eumelanin light absorption ranges aver the whole visible spectrum but is lower for higher wavelengths, we can take advantage from the Nd:YAG laser, using infrared $(1064 \mathrm{~nm})$ radiation in order to reduce the risk of damaging molecules during target ablation.

Therefore eumelanin thin films $[43,44]$ have been successfully deposited with MAPLE technique from a frozen water suspension using infrared laser radiation even if the low laser absorption of ice together with the high absorption of eumelanin suggests that the target ablation is due to laser energy absorbed by the eumelanin molecules, followed by thermal energy transfer and ejection of ice/water/vapour containing undamaged eumelanin molecules and supramolecular structures.

Due to the presence of supramolecular structures, eumelanin films show some surface roughness (Figure 9). Changing deposition parameters (e.g., reducing deposition rate by reducing laser pulse repetition rate) reduces surface roughness. On the contrary, deposition on heated substrates increases film roughness (Figure 10) while postannealing is ineffective [44]. 


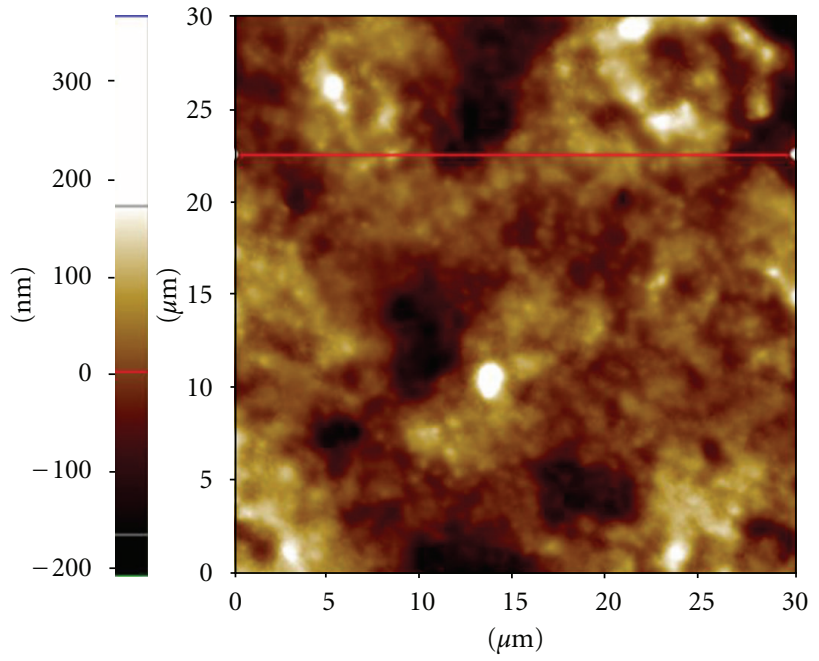

(a)

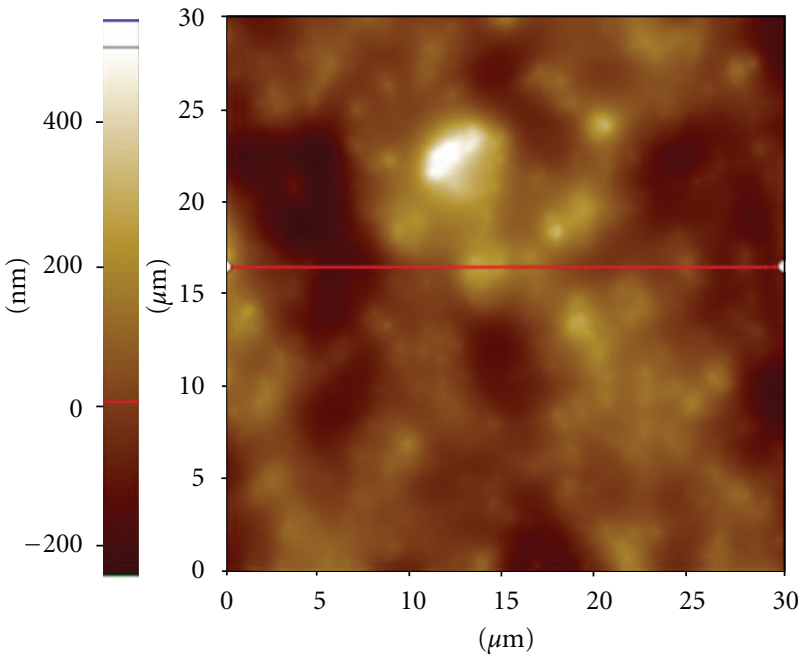

(c)

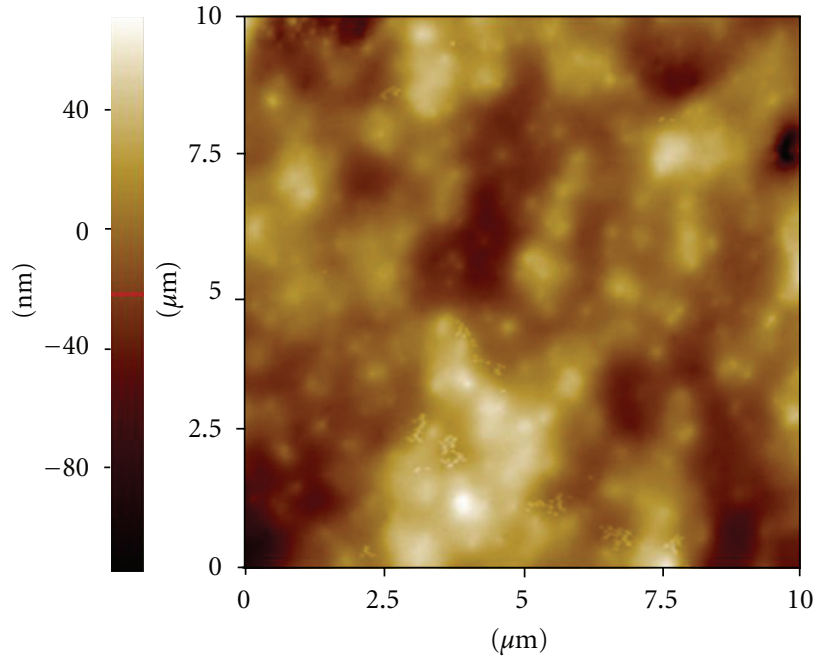

(b)

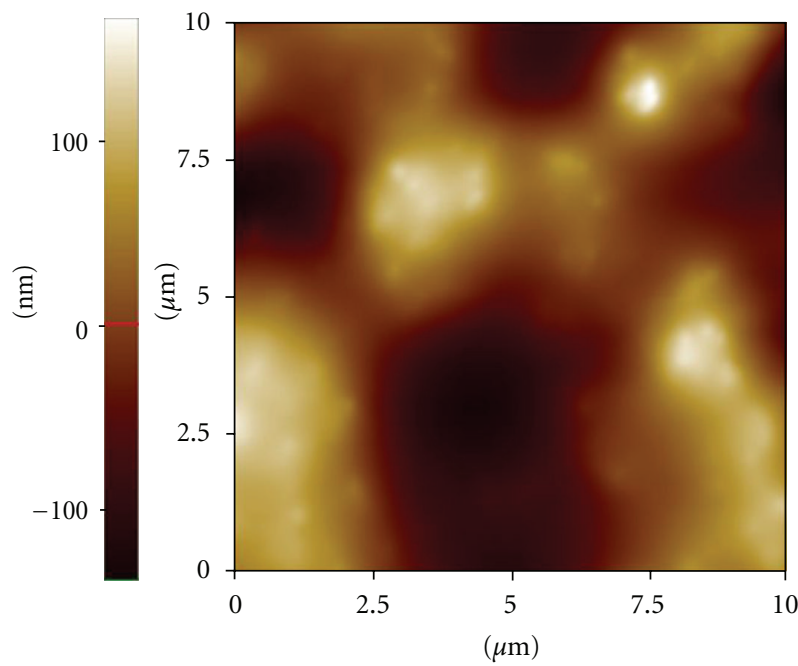

(d)

Figure 9: AFM images of two melanin films obtained with different pulse repetition rates (a) and (b): 4 pulse/s; (c) and (d): 2 pulse/s but the same total number of pulses (10000 pulses).

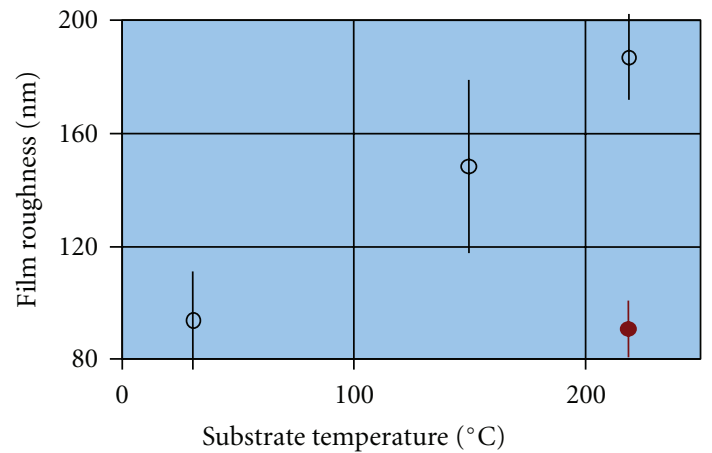

FIGURE 10: Surface roughness, given as RMS values, of eumelanin films deposited with different substrate temperatures (void circles). Another sample (filled circle) has been deposits on substrate at $32^{\circ} \mathrm{C}$ followed by in situ annealing at $220^{\circ} \mathrm{C}$.
Preliminary electrical characterization shows that current-voltage plots seem to obey Ohm's law without evidence of the to be affected by the presence of significant energy barriers, charge transport being drastically reduced in vacuum, even if the phenomenon is at least partially reversible.

\section{Conclusions and Future Applications}

A lot of effort is currently devoted to the study of devices using organic or biological materials and nanostructures. In most cases the deposition of thin or multilayered films is required. In most cases PLD fails in depositing such soft materials damaging them during ablation. On the contrary MAPLE is well suited as a laser deposition technique capable to avoid structural damages to the material of interest. 
The experimental results obtained show that MAPLE deposition technique can take advantage from the use of a Nd:YAG laser equipped with second and third harmonic generation (SHG/THG) devices (wavelengths $1064 \mathrm{~nm}, 532 \mathrm{~nm}$, and $355 \mathrm{~nm}$ ) in order to (i) produce multilayered film structures, (ii) add surface layers to three-dimensional porous structure partially maintaining its porosity, and (iii) grow thin film starting from a suspension of light absorbing molecular aggregates.

Our experiments have shown that MAPLE applicability goes beyond classical situations (thin film deposition obtained from light transparent molecules highly soluble in light absorbing solvent) but can be used for deposition of soft materials on tridimensional structures, of poorly soluble guest molecules and of light absorbing molecules.

Of special interest is the possibility to obtain eumelanin thin films. Given the capability of melanin molecules to interact with metallic ions, we think that the charge transport properties of doped eumelanin thin films must be investigated in view of the realization of innovative devices.

It is also clear that the deposition layout (solvent choice, solute concentration, substrate position, and orientation) and parameters (laser wavelength, beam pulse repetition rate, pulse duration, substrate temperature) are critical in avoiding cluster creation during deposition and reducing film roughness. Some experimental and theoretical effort must be actually devoted to reach a fully understanding of the MAPLE deposition mechanism.

\section{Acknowledgments}

Financial support from EU FP7 project MAMA Grant Agreement no. 264098 and from the regional project FARO Fabrication of Organic Transistors for Bio-Sensing Applications is gratefully acknowledged.

\section{References}

[1] A. Piqué, R. A. McGill, D. B. Chrisey et al., "Growth of organic thin films by the matrix assisted pulsed laser evaporation (MAPLE) technique," Thin Solid Films, vol. 355-356, pp. 536$541,1999$.

[2] R. Eason, Ed., Pulsed Laser Deposition of Thin Films, John Wiley \& Sons, New York, NY, USA, 2007.

[3] D. B. Chrisey, A. Piqué, R. A. McGill et al., "Laser deposition of polymer and biomaterial films," Chemical Reviews, vol. 103, no. 2, pp. 553-576, 2003.

[4] A. Gutierrez-Llorente, R. Perez-Casero, B. Pajot et al., "Growth of anthracene thin films by matrix-assisted pulsed-laser evaporation," Applied Physics A, vol. 77, no. 6, pp. 785-788, 2003.

[5] R. Cristescu, D. Mihaiescu, G. Socol, I. Stamatin, I. N. Mihailescu, and D. B. Chrisey, "Deposition of biopolymer thin films by matrix assisted pulsed laser evaporation," Applied Physics A, vol. 79, no. 4-6, pp. 1023-1026, 2004.

[6] R. Fryček, M. Jelínek, T. Kocourek et al., "Thin organic layers prepared by MAPLE for gas sensor application," Thin Solid Films, vol. 495, no. 1-2, pp. 308-311, 2006.

[7] K. Rodrigo, P. Czuba, B. Toftmann, J. Schou, and R. Pedrys, "Surface morphology of polyethylene glycol films produced by matrix-assisted pulsed laser evaporation (MAPLE): dependence on substrate temperature," Applied Surface Science, vol. 252, no. 13, pp. 4824-4828, 2006.

[8] E. Leveugle and L. V. Zhigilei, "Molecular dynamics simulation study of the ejection and transport of polymer molecules in matrix-assisted pulsed laser evaporation," Journal of Applied Physics, vol. 102, no. 7, Article ID 074914, 19 pages, 2007.

[9] A. Sellinger, E. Leveugle, J. M. Fitz-Gerald, and L. V. Zhigilei, "Generation of surface features in films deposited by matrixassisted pulsed laser evaporation: the effects of the stress confinement and droplet landing velocity," Applied Physics A, vol. 92, no. 4, pp. 821-829, 2008.

[10] A. Piqué, P. Wu, B. R. Ringeisen et al., "Processing of functional polymers and organic thin films by the matrix-assisted pulsed laser evaporation (MAPLE) technique," Applied Surface Science, vol. 186, no. 1-4, pp. 408-415, 2002.

[11] V. Califano, F. Bloisi, L. R. M. Vicari, D. M. Yunos, X. Chatzistavrou, and A. R. Boccaccini, "Matrix assisted pulsed laser evaporation (MAPLE) of poly(D,L lactide) (PDLLA) on three dimensional bioglass structures," Advanced Engineering Materials, vol. 11, no. 8, pp. 685-689, 2009.

[12] C. N. Hunter, M. H. Check, J. E. Bultman, and A. A. Voevodin, "Development of matrix-assisted pulsed laser evaporation (MAPLE) for deposition of disperse films of carbon nanoparticles and gold/nanoparticle composite films," Surface and Coatings Technology, vol. 203, no. 3-4, pp. 300-306, 2008.

[13] A. P. Caricato, A. Luches, and R. Rella, "Nanoparticle thin films for gas sensors prepared by matrix assisted pulsed laser evaporation," Sensors, vol. 9, no. 4, pp. 2682-2696, 2009.

[14] T. Smausz, G. Megyeri, R. Kékesi et al., "Comparative study on pulsed laser deposition and matrix assisted pulsed laser evaporation of urease thin films," Thin Solid Films, vol. 517, no. 15, pp. 4299-4302, 2009.

[15] E. J. Houser, D. B. Chrisey, M. Bercu et al., "Functionalized polysiloxane thin films deposited by matrix-assisted pulsed laser evaporation for advanced chemical sensor applications," Applied Surface Science, vol. 252, no. 13, pp. 4871-4876, 2006.

[16] F. Bloisi, L. Vicari, R. Papa et al., "Biomaterial thin film deposition and characterization by means of MAPLE technique," Materials Science and Engineering C, vol. 27, no. 5-8, pp. 11851190, 2007.

[17] A. P. Caricato, M. Lomascolo, A. Luches et al., "MAPLE deposition of methoxy Ge triphenylcorrole thin films," Applied Physics A, vol. 93, no. 3, pp. 651-654, 2008.

[18] V. Califano, F. Bloisi, L. R. Vicari, O. Bretcanu, and A. R. Boccaccini, "Matrix-assisted pulsed laser evaporation of poly(D,L-lactide) for biomedical applications: effect of near infrared radiation," Journal of Biomedical Optics, vol. 13, no. 1, Article ID 014028, 2008.

[19] E. Leveugle, L. V. Zhigilei, A. Sellinger, and J. M. Fitz-Gerald, "Computational and experimental study of the cluster size distribution in MAPLE," Applied Surface Science, vol. 253, no. 15, pp. 6456-6460, 2007.

[20] D. M. Bubb, A. O. Sezer, J. Gripenburg, B. Collins, and E. Brookes, "Assessing the effect of the matrix in resonant infrared MAPLE," Applied Surface Science, vol. 253, no. 15, pp. 6465-6470, 2007.

[21] V. Califano, F. Bloisi, L. Vicari et al., "Dependence on substrate temperature of the conformation and structure of a poly[3-(4octyloxyphenyl)thiophene] (POOPT) thin film obtained by matrix assisted pulsed laser evaporation (MAPLE)," Physica Status Solidi A, vol. 206, no. 9, pp. 2166-2170, 2009.

[22] A. P. Caricato, G. Leggieri, M. Martino et al., "Dependence of the surface roughness of MAPLE-deposited films on the 
solvent parameters," Applied Physics A, vol. 101, no. 4, pp. 759$764,2010$.

[23] A. Luches and A. P. Caricato, "Matrix assisted pulsed laser evaporation: the surface cluster problem," Applied Physics B, vol. 105, no. 3, pp. 503-508, 2011.

[24] F. Bloisi, A. Cassinese, R. Papa, L. Vicari, and V. Califano, "Matrix-assisted pulsed laser evaporation of polythiophene films," Thin Solid Films, vol. 516, no. 7, pp. 1594-1598, 2008.

[25] A. T. Sellinger, E. M. Leveugle, K. Gogick, L. V. Zhigilei, and J. M. Fitz-Gerald, "Laser processing of polymer nanocomposite thin films," Journal of Vacuum Science and Technology A, vol. 24, no. 4, pp. 1618-1622, 2006.

[26] A. T. Sellinger, E. Leveugle, K. Gogick, G. Peman, L. V. Zhigilei, and J. M. Fitz-Gerald, "Ejection of matrix-polymer clusters in matrix-assisted laser evaporation: experimental observations," Journal of Physics, vol. 59, no. 1, pp. 314-317, 2007.

[27] R. O. Esenaliev, A. A. Karabutov, N. B. Podymova, and V. S. Letokhov, "Laser ablation of aqueous solutions with spatially homogeneous and heterogeneous absorption," Applied Physics $B$, vol. 59, no. 1, pp. 73-81, 1994.

[28] A. Piqué, R. C. Y. Auyeung, J. L. Stepnowski et al., "Laser processing of polymer thin films for chemical sensor applications," Surface and Coatings Technology, vol. 163-164, pp. 293 299, 2003.

[29] J. M. Fitz-Gerald, G. Jennings, R. Johnson, and C. L. Fraser, "Matrix assisted pulsed laser deposition of light emitting polymer thin films," Applied Physics A, vol. 80, no. 5, pp. 11091112, 2005.

[30] B. R. Ringeisen, J. Callahan, P. K. Wu et al., "Novel laser-based deposition of active protein thin films," Langmuir, vol. 17, no. 11, pp. 3472-3479, 2001.

[31] L. Stamatin, R. Cristescu, G. Socol et al., "Laser deposition of fibrinogen blood proteins thin films by matrix assisted pulsed laser evaporation," Applied Surface Science, vol. 248, no. 1-4, pp. 422-427, 2005.

[32] A. Purice, J. Schou, P. Kingshott, and M. Dinescu, "Production of active lysozyme films by matrix assisted pulsed laser evaporation at $355 \mathrm{~nm}$," Chemical Physics Letters, vol. 435, no. 4-6, pp. 350-353, 2007.

[33] A. Purice, J. Schou, P. Kingshott, N. Pryds, and M. Dinescu, "Characterization of lysozyme films produced by matrix assisted pulsed laser evaporation (MAPLE)," Applied Surface Science, vol. 253, no. 15, pp. 6451-6455, 2007.

[34] P. K. Wu, B. R. Ringeisen, D. B. Krizman et al., "Laser transfer of biomaterials: matrix-assisted pulsed laser evaporation (MAPLE) and MAPLE direct write," Review of Scientific Instruments, vol. 74, no. 4, pp. 2546-2557, 2003.

[35] A. P. Caricato, M. G. Manera, M. Martino et al., "Uniform thin films of $\mathrm{TiO}_{2}$ nanoparticles deposited by matrix-assisted pulsed laser evaporation," Applied Surface Science, vol. 253, no. 15, pp. 6471-6475, 2007.

[36] D. M. Bubb, P. K. Wu, J. S. Horowitz et al., "The effect of the matrix on film properties in matrix-assisted pulsed laser evaporation," Journal of Applied Physics, vol. 91, no. 4, pp. 2055-2058, 2002.

[37] V. Califano, F. Bloisi, L. R. M. Vicari, P. Colombi, E. Bontempi, and L. E. Depero, "MAPLE deposition of biomaterial multilayers," Applied Surface Science, vol. 254, no. 22, pp. 7143-7148, 2008.

[38] L. L. Hench, R. J. Splinter, W. C. Allen, and T. K. Greenlee, "Bonding mechanisms at the interface of ceramic prosthetic materials," Journal of Biomedical Materials Research, vol. 5, no. 6, pp. 117-141, 1971.
[39] Q. Z. Chen, I. D. Thomson, and A. R. Boccaccini, "45S5 Bioglass-derived glass-ceramic scaffolds for bone tissue engineering," Biomaterials, vol. 27, no. 11, pp. 2414-2425, 2006.

[40] A. Roviello, A. Buono, A. Carella et al., "Regioregular poly[3(4-alkoxyphenyl)thiophene]s," Journal of Polymer Science A, vol. 45, no. 9, pp. 1758-1770, 2007.

[41] M. Barra, A. C. Barone, M. Biasiucci et al., "Direct current and alternating current electrical transport properties of regioregular poly[3-(4-alkoxyphenyl)-thiophenes]," Journal of Applied Physics, vol. 102, no. 9, Article ID 093712, 7 pages, 2007.

[42] V. Califano, F. Bloisi, L. Vicari et al., "Substrate temperature dependence of the structure of polythiophene thin films obtained by matrix assisted pulsed laser evaporation (MAPLE)," The European Physical Journal Applied Physics, vol. 48, no. 1, pp. 10505-10512, 2009.

[43] F. Bloisi, A. Pezzella, M. Barra, F. Chiarella, A. Cassinese, and L. Vicari, "Matrix assisted pulsed laser deposition of melanin thin films," Journal of Applied Physics, vol. 110, no. 2, Article ID 026105, 3 pages, 2011.

[44] F. Bloisi, A. Pezzella, M. Barra et al., "Effect of substrate temperature on MAPLE deposition of synthetic eumelanin films," Applied Physics A, vol. 105, no. 3, pp. 619-627, 2011. 

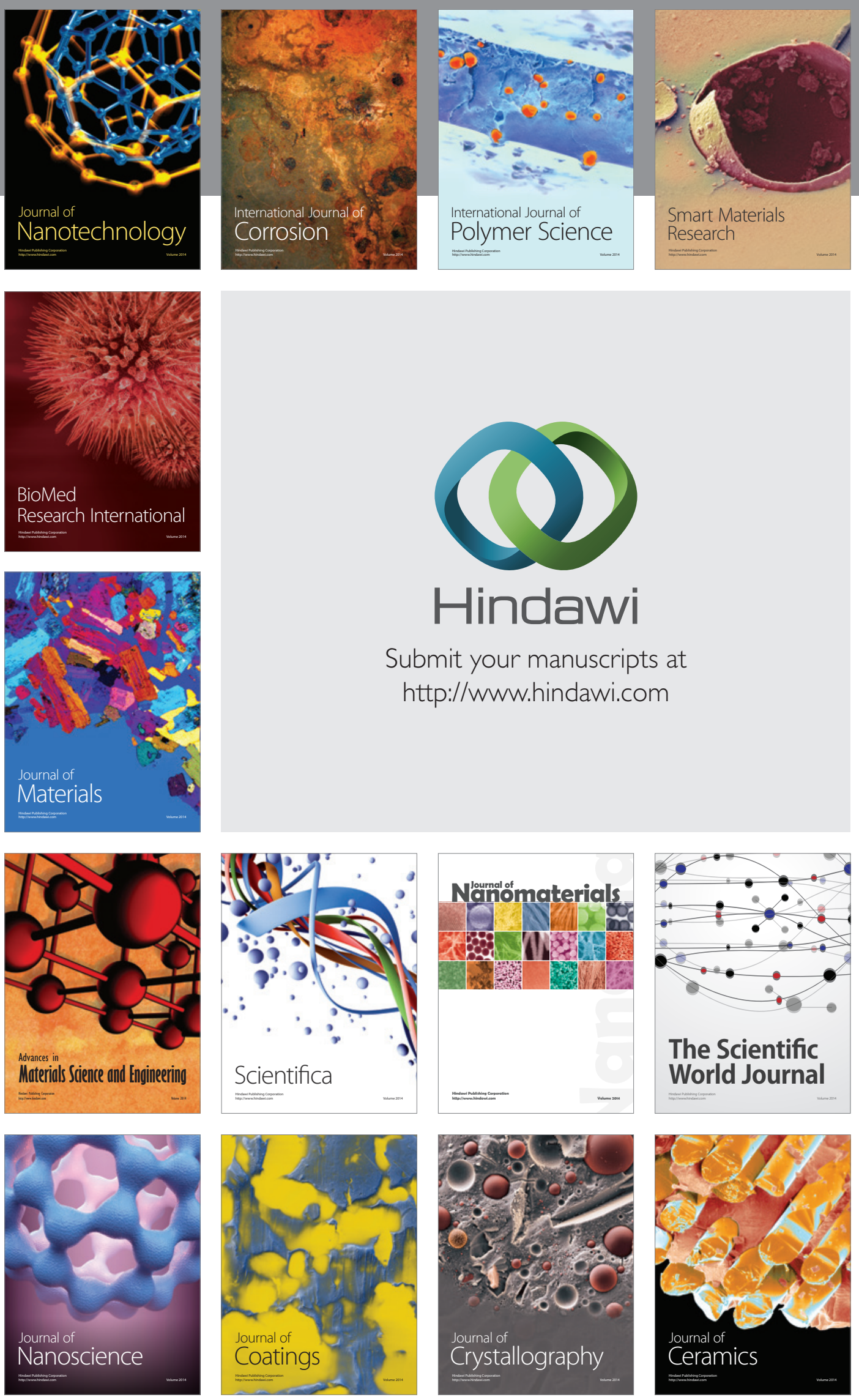

The Scientific World Journal

Submit your manuscripts at

http://www.hindawi.com

\section{World Journal}

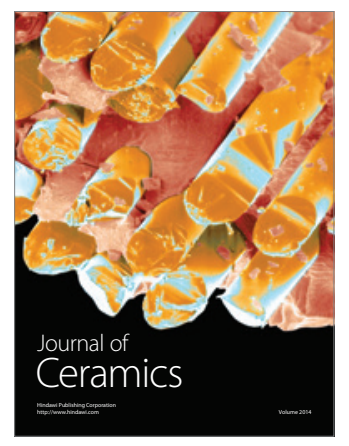

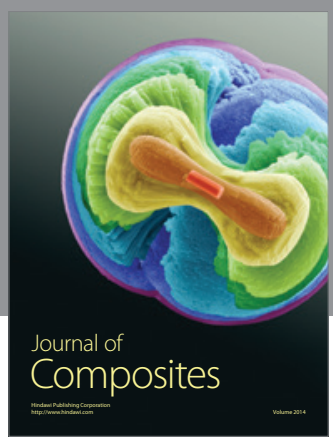
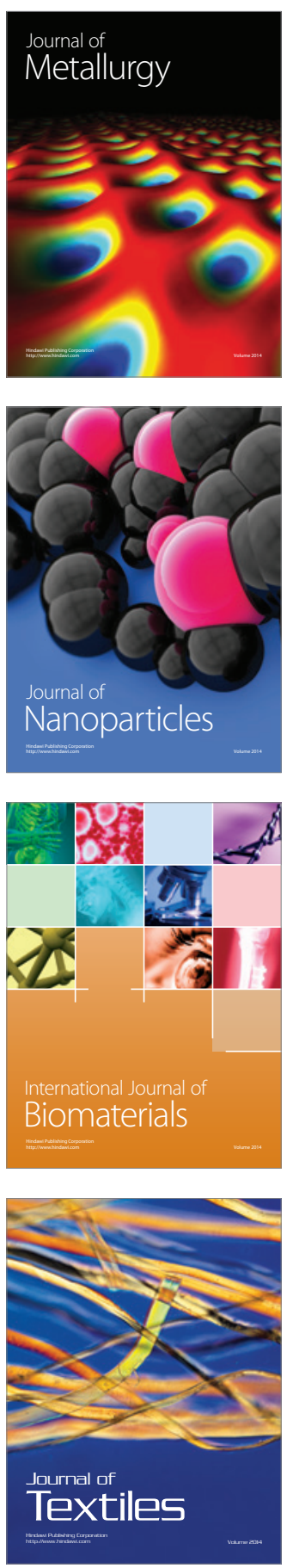